\title{
ANALYTIC CONTINUATION OF THE PRINCIPAL SERIES
}

\author{
BY R. A. KUNZE AND E. M. STEIN ${ }^{1}$
}

Communicated by Edwin Hewitt, July 22, 1961

The purpose of this note is to announce results obtained in the analytic continuation of the (nondegenerate) "principal series" of representations of the $n \times n$ complex unimodular group. This study has as its starting point a similar one for the $2 \times 2$ real unimodular group previously carried out by us in [4].

We let $G$ be the $n \times n$ complex unimodular group and $C$ its diagonal subgroup consisting of elements $c=\left(c_{1}, c_{2}, \cdots, c_{n}\right)$. A continuous character $\lambda$ of $C$ is given by

$$
\lambda(c)=\left(\frac{c_{1}}{\left|c_{1}\right|}\right)^{m_{1}} \cdots\left(\frac{c_{n}}{\left|c_{n}\right|}\right)^{m_{n}}\left|c_{1}\right|^{s_{1}} \cdots\left|c_{n}\right|^{s_{n}}
$$

where the sequences of integers $m_{1}, m_{2}, \cdots, m_{n}$ and complex numbers $s_{1}, s_{2}, \cdots, s_{n}$ are uniquely determined by setting $0 \leqq m_{1}+m_{2}$ $+\cdots+m_{n}<n$ and $s_{1}+s_{2}+\cdots+s_{n}=0$. Notice that $\lambda$ is unitary, i.e., has values in the circle group, if $\operatorname{Re}\left(s_{j}\right)=0, j=1,2, \cdots, n$. Gelfand and Neumark have shown how to construct for each unitary $\lambda$ an irreducible unitary representation $a \rightarrow T(a, \lambda)$ of the group $G$ [2]. To describe these representations (i.e., the principal series) we follow the method but not the notation of [2].

Let $V$ be the subgroup of $G$ of elements having ones on the main diagonal and zeros above the main diagonal. Then $G$ acts on $V$ in a natural way; we denote the action of $a \in G$ on $v \in V$ by $v \tilde{a}$ (the transformations $v \rightarrow v \tilde{a}$ are linear fractional transformations when $n=2$ and generalizations thereof in higher dimensions). The operators of the representation $T(\cdot, \lambda)$ are given by

$$
T(a, \lambda) f(v)=m(v, a ; \lambda) f(v \tilde{a})
$$

where $m(v, a ; \lambda)$ is an appropriate multiplier, and the underlying Hilbert space is $L_{2}(V)$.

In order to state our results we introduce a tube $J$ lying in the complex hyperplane $s_{1}+s_{2}+\cdots+s_{n}=0$. The base $B$ of $J$ is the smallest convex set which contains the points $(\sigma,-\sigma, 0,0, \cdots, 0)$, $-1<\sigma<1$ and is invariant under all permutations of coordinates. A

\footnotetext{
1 This work was supported in part by the National Science Foundation-Research Grant NSF G 12847.
} 
point $\left(s_{1}, s_{2}, \cdots, s_{n}\right), s_{j}=\sigma_{j}+i t_{j}$ belongs to $J$ if and only if $s_{1}+s_{2}+$ $\cdots+s_{n}=0$ and $\left(\sigma_{1}, \sigma_{2}, \cdots, \sigma_{n}\right) \in B$.

THEOREM 1. For each character $\lambda=\lambda\left(m_{1}, m_{2}, \cdots, m_{n} ; s_{1}, s_{2}, \cdots, s_{n}\right)$ for which $\left(s_{1}, s_{2}, \cdots, s_{n}\right) \in J$ there exists a representation $a \rightarrow R(a, \lambda)$ of $G$ on $L_{2}(V)$. The family of all such representations has the following properties:

(1) For each fixed $\lambda, a \rightarrow R(a, \lambda)$ is a continuous uniformly bounded representation.

(2) For fixed $a, R(a, \lambda)$ as a function of $\left(s_{1}, s_{2}, \cdots, s_{n}\right)$ is analytic in the tube $\mathrm{J}$.

(3) When $\lambda$ is unitary, $R(\cdot, \lambda)$ is unitarily equivalent to $T(\cdot, \lambda)$, the corresponding member of the principal series.

(4) $R(\cdot, \lambda)=R(\cdot, p \lambda)$ for every "permutation" $p$ of the character $\lambda$.

(5) $R(\cdot, \lambda)^{\prime}=R\left(\cdot, \lambda^{\prime}\right)$ where $R(a, \lambda)^{\prime}=R\left(a^{-1}, \lambda\right)^{*}$ and $\lambda^{\prime}(c)=\bar{\lambda}\left(c^{-1}\right)$.

In their work on the principal series Gelfand and Neumark obtained a trace formula for certain operators associated with the representations $a \rightarrow T(a, \lambda),[2$, p. 73]. The formula involves a function (the character of the representation) which we denote by $\psi(a, \lambda)$. It is initially defined for unitary characters $\lambda$ but extends in an obvious way by analyticity to nonunitary characters. We prove the following result.

THEOREM 2. Let $\lambda$ be as in Theorem 1 and $f$ be the convolution of two bounded functions on $G$ with compact support. Then the operator

$$
R(f, \lambda)=\int_{G} f(a) R(a, \lambda) d a
$$

is of trace class, and

$$
\operatorname{tr} R(f, \lambda)=\int_{G} f(a) \psi(a, \lambda) d a .
$$

REMARKs. (i) It is known that $T(\cdot, \lambda)$ and $T(\cdot, p \lambda)$ are unitarily equivalent for every permutation $p[2, \mathrm{p} .118]$. In the present context this unitary equivalence becomes an identity by (4) of Theorem 1.

(ii) It follows almost immediately from (4) and (5) that $R(\cdot, \lambda)$ is unitary if there exists a permutation $p$ such that $\lambda^{\prime}=p \lambda$, where $\lambda^{\prime}$ is the contragredient of $\lambda$ defined by $\lambda^{\prime}(c)=\bar{\lambda}\left(c^{-1}\right)$. If $\lambda^{\prime}=p \lambda$ and $p$ equals the identity we are dealing with the principal series. However, when $p$ is not the identity and $\lambda^{\prime}=p \lambda$, it then follows from Theorem 2 that $R(\cdot, \lambda)$ is unitarily equivalent to a member of the complementary series [3]. 
(iii) By means of Theorem 2 we also show that if $\lambda^{\prime} \neq p \lambda$ for all $p$, then the representation $R(\cdot, \lambda)$ is not equivalent to a unitary one, although it is uniformly bounded. The existence of a group $(S L(2, R))$ and uniformly bounded representations of it not equivalent to unitary ones was first proved by Ehrenpreis and Mautner [1].

We shall briefly describe the ideas behind the proofs of Theorems 1 and 2 . We begin by limiting our attention to unitary characters $\lambda$. A basic fact we prove can be stated as follows:

LEMMA. Let $G_{0}$ be the subgroup of $G$ consisting of the matrices a such that $a_{j n}=0,1 \leqq j \leqq n-1$. For any character $\lambda$, call the integer $r$, uniquely determined by $0 \leqq r<n, r=m_{1}+m_{2}+\cdots+m_{n}$, the residue of the character $\lambda$. Then if $\lambda_{1}$ and $\lambda_{2}$ have the same residue the restrictions of $T\left(\cdot, \lambda_{1}\right)$ and $T\left(\cdot, \lambda_{2}\right)$ to $G_{0}$ are unitarily equivalent.

Thus when we restrict to $G_{0}$, there are only $n$ (essentially) different representations among the principal series. This is remarkable in view of the known fact that the members of the principal series are all irreducible on $G_{0}[2$, p. 22]. Hence there is a unitary operator $W(\lambda)$ so that if we set

$$
R(a, \lambda)=W(\lambda) T(a, \lambda) W^{-1}(\lambda)
$$

then $R(a, \lambda)$ for fixed $a \in G_{0}$ depends only on the residue of $\lambda$. We call the representations $R(\cdot, \lambda)$ the normalized principal series. It is these that can be continued analytically (i.e., to nonunitary $\lambda$ 's) as in Theorem 1 , while the $T(\cdot, \lambda)$ cannot.

The actual construction of the operators $W(\lambda)$ is too complicated to describe here but is intimately connected with the construction of the intertwining operators $A(p, \lambda)$ between $T(\cdot, \lambda)$ and $T(\cdot, p \lambda)$. In fact, it follows from part (4) of Theorem 1 that up to a constant multiple

$$
A(p, \lambda)=W^{-1}(p \lambda) W(\lambda) .
$$

Moreover, we show that the operator $W(\lambda)$ can be written as a product of $n(n-1) / 2$ operators of the type $A(p, \lambda)$.

The work described above has many points of contact with the analysis of the special case of the $2 \times 2$ (real) group which we carried out previously in [4]. However, there is an essential difference. This is due to the fact that the study of the representations in question is closely related to the Fourier analysis on $L_{2}(V)$. When $n=2, V$ is a commutative group; but this is not so when $n>2$, and therein lies the major obstacle to the proof of Theorem 1. 


\section{REFERENCES}

1. L. Ehrenpreis and F. I. Mautner, Uniformly bounded representations of groups, Proc. Nat. Acad. Sci. U.S.A. vol. 41 (1955) pp. 231-233.

2. I. M. Gelfand and M. A. Neumark, Unitäre Darstellungen der klassischen Gruppen, Berlin, Akademie-Verlag, 1957.

3. Harish-Chandra, Representations of semi-simple Lie groups. III, Trans. Amer. Math. Soc. vol. 76 (1954) pp. 234-253.

4. R. A. Kunze and E. M. Stein, Uniformly bounded representations and harmonic analysis of the $2 \times 2$ real unimodular group, Amer. J. Math. vol. 82 (1960) pp. 1-62.

BRANDEIS UNIVERSITY AND

The UnIVersity of Chicago 